\title{
FREQUÊNCIA CARDÍACA PARA ESTIMATIVAS DA CARGA FÍSICA DE TRABALHO NA EXPLORAÇÃO FLORESTAL
}

\author{
HEART RATE FOR ESTIMATES THE PHYSICAL WORKLOAD IN FOREST LOGGING
}

\author{
Kauê Augusto Oliveira Nascimento ${ }^{1}$, Niro Higuchi' ${ }^{2}$, Fabiano Emmert ${ }^{3}$ \\ ${ }^{1}$ Universidade Federal do Paraná, Curitiba, Paraná, Brasil - kaue_nascimento@hotmail.com \\ ${ }^{2}$ Instituto Nacional de Pesquisas da Amazônia, Manaus, Amazonas, Brasil-higuchi.niro@gmail.com \\ ${ }^{3}$ Universidade Federal Rural da Amazônia, Belém, Pará, Brasil-fabianoemmert@yahoo.com.br
}

\section{RESUMO}

O trabalho em exploração florestal exige grande contingente e carga física sob condições ambientais desfavoráveis. Portanto, um bom condicionamento e hábitos saudáveis são essenciais para o desempenho. $O$ estudo pretendeu a coleta de frequência cardíaca para estimar a carga física do trabalho em exploração florestal. A pesquisa foi conduzida no município de Itapiranga, Amazonas, Brasil. Foram avaliadas as operações de pré-arraste, arraste e pátio, exceto o corte. Foi coletada frequência cardíaca da ocupação mais desgastante em cada operação, e estimadas as cargas físicas por dois métodos: anexo C da ISO 8996 e o método CCV. A variável peso demonstrou forte correlação positiva com a frequência cardíaca. A carga física variou em até $167 \mathrm{kcal} / \mathrm{h}$ para a mesma ocupação. A ocupação puxador de cabo (pré-arraste) foi a mais desgastante, sendo considerada pesada e estimou-se pausas de até $15 \mathrm{~min} . / \mathrm{h}$. A frequência cardíaca demonstrou ser indicadora do condicionamento físico, um aspecto importante, pois interfere no desempenho do trabalhador.

PALAVRAS-CHAVE: Amazônia, Gasto metabólico, Manejo florestal.

\section{ABSTRACT}

The work in forest logging requires large contingent and physical load under unfavorable environmental thermal conditions. Therefore, physical shape and healthy habits are essential for performance. The study aimed at the collection of heart rate to estimate the physical workload in logging. The study was conducted in the municipality of Itapiranga, Amazonas, Brazil. The operations were divided on: cutting, pre-drag, drag and patio; with the exception of cutting all were evaluated. Heart rate was collected from the most exhausting occupation in each operation, and with this, the physical loads were estimated by two methods: Annex C of ISO 8996 and the CCV method. The weight variable showed a positive correlation with the heart rate. The physical load varied up to $167 \mathrm{kcal} / \mathrm{h}$ in the same occupation. The cable pull occupation (pre-drag) was the most exhausting and considered heavy, and pauses of up to $15 \mathrm{~min} / \mathrm{h}$ were calculated. Heart rate estimation resulted in a physical conditioning indicator that interferes with work performance.

KEY-WORDS: Amazon, Forest management, Metabolic rate. 


\section{INTRODUÇÃO}

A Amazônia é o maior bioma do Brasil e um dos mais diversos em espécies vegetais no mundo. Abrange uma área de cerca de $50 \%$ do território brasileiro, onde a maior parte é constituída de Florestas de Terra Firme, ainda pouco manejadas. O setor madeireiro é importante para a economia da região e possui grande perspectiva de crescimento, por tratar-se de uma área extensa com grande estoque de madeira (HIGUCHI et al., 2007).

O trabalho neste setor é considerado um dos mais extenuantes e perigosos no mundo em comparação a outras atividades econômicas (HARSTELA, 1990; HAGEN et al., 1993; LILLEY et al., 2002). Lima \& Leite (2014) destaca que nos países industrializados, a mecanização já chegou a um nível tão alto que o trabalho mais pesado é feito em rodízio por diversos trabalhadores, porém no Brasil isso ainda não é uma realidade, atividade em que ainda há baixa mecanização e grande demanda por mão de obra nas operações florestais (SANT'ANNA \& MALINOVSKI, 2002; LIMA \& LEITE, 2014). Atrelado a isso, as operações que envolvem o manejo florestal na região amazônica demandam grande número de trabalhadores em todas as etapas da cadeia produtiva (SABOGAL et al., 2006).

Emmert (2014) destaca que em exploração seletiva de madeira em florestas tropicais, o trabalho exige grande contingente e alta carga física, em uma floresta com denso sub-bosque e sob condições térmicas ambientais desfavoráveis ao trabalho. Em razão destas condições específicas, um bom condicionamento físico e hábitos saudáveis são essenciais para um trabalhador ser produtivo nesta atividade (HARSTELA, 1990; HAGEN et al., 1993; SANT'ANNA \& MALINOVSKI, 2002), o que justifica a realização deste trabalho.

Este trabalho propõe as hipóteses que as variáveis frequência cardíaca, peso, e idade predizem bem a carga física do trabalho florestal na Amazônia, correlacionadas ao condicionamento físico, importante para a saúde, segurança e produtividade das operações. O mesmo teve como objetivo a coleta de variáveis antropométricas e frequência cardíaca dos trabalhadores para estimativa da carga física do trabalho em exploração florestal na Amazônia, com uso de dois métodos diferentes.

\section{MATERIAL E MÉTODOS}

\section{Área de estudo}

O trabalho foi desenvolvido em uma empresa que executa manejo florestal seletivo em área privada, localizada no estado do Amazonas, Brasil. O estudo foi conduzido na unidade de produção anual (UPA) localizada no município de Itapiranga, coordenadas latitude $02^{\circ} 75^{\prime}$ $\mathrm{S}$ e longitude $58^{\circ} 47^{\prime} \mathrm{W}$. O clima na área é classificado como tropical úmido (Af), com precipitação média anual de $2200 \mathrm{~mm}$ e sem estação seca definida de acordo com a classificação de Koppen-Geiger (ALVARES et al., 2013). A temperatura média é de $28^{\circ} \mathrm{C}$ e umidade relativa do ar de $80 \%$ (INMET, 2016). A fitofisionomia da região é Floresta de Terra Firme, dominante na paisagem amazônica (LEITÃO FILHO, 1987).

A exploração florestal utiliza sistema de toras longas, em que é usada a motosserra para o corte, trator com guincho por cabo de aço para o pré-arraste até a beira do ramal e máquina Skidder para arraste das toras até os pátios (BATISTA, 2008; EMMERT, 2014). As etapas da exploração são divididas em: corte, pré-arraste, arraste. Para as análises foram consideradas também as operações de pátio.

\section{Coleta de dados}

Foram analisados 06 trabalhadores do sexo masculino na ocupação mais desgastante dentro de cada uma das operações: pré-arraste (04), arraste (01) e pátio (01). A coleta de dados foi realizada durante o período diurno no mês de novembro de 2015. A escolha das equipes analisadas foi realizada por sorteio. O esforço amostral consta na Tabela 1.

Tabela 1. Ocupações avaliadas e esforço de coleta da frequência cardíaca

\begin{tabular}{ccccc}
\hline $\begin{array}{c}\text { Etapa } \\
\text { analisada }\end{array}$ & $\begin{array}{c}\text { Ocupação } \\
\text { avaliada }\end{array}$ & $\begin{array}{c}\text { No turnos } \\
\text { avaliados }\end{array}$ & $\begin{array}{c}\text { Horas } \\
\text { coleta }\end{array}$ & $\begin{array}{c}\text { Número } \\
\text { amostras }\end{array}$ \\
\hline $\begin{array}{c}\text { Pré- } \\
\text { arraste }\end{array}$ & $\begin{array}{c}\text { Puxador de } \\
\text { cabo }\end{array}$ & 04 & 44 & $>6336$ \\
Arraste & $\begin{array}{c}\text { Líder de } \\
\text { equipe }\end{array}$ & 01 & 11 & $>1584$ \\
$\begin{array}{c}\text { Pátio } \\
\text { principal }\end{array}$ & $\begin{array}{c}\text { Operador de } \\
\text { carregadeira }\end{array}$ & 01 & 12 & $>1728$ \\
\hline
\end{tabular}

Foi utilizado um medidor de frequência cardíaca Garmin modelo FR70, com monitor cardíaco de pulso e cinta para acoplar ao peito, com opções para adição de informações pessoais: peso, idade, gênero e preparo físico. O limite de erro nas estimativas do equipamento é de $10 \%$ para um período de coleta de 1 hora (GARMIN, 2015). A frequência cardíaca dos trabalhadores foi registrada pelo equipamento a cada 25 segundos durante 
toda a jornada, incluindo as paradas para descanso e almoço. Ressalta-se que este aparelho foi desenvolvido para fins desportivos (corrida), no entanto, demonstrou ser adequado para avaliação ocupacional, desde que sejam evitados longos períodos de mensuração, devido a limitações de memória. Sanches (2012) utilizou este tipo de equipamento em seu estudo na colheita de madeira e obteve resultados satisfatórios.

Foi utilizado método proposto no anexo $\mathrm{C}$ da norma ISO 8996 (Ergonomia térmica do ambiente Determinação da taxa de calor metabólico), que trata da classificação de taxa metabólica por meio de variáveis pessoais e é muito utilizada em avaliações ocupacionais. Foi utilizado o valor médio de frequência cardíaca (bpm) em cada jornada, além da coleta de variáveis pessoais de cada trabalhador. A partir disso, foi possível a estimativa da carga física de trabalho em $\mathrm{W} / \mathrm{m}^{2}$, pela aplicação das fórmulas presente na Tabela 2.

Tabela 2. Fórmulas para conversão de frequência cardíaca (HR) em taxa metabólica $\left(\mathrm{W} / \mathrm{m}^{2}\right)$ presentes no anexo $\mathrm{C}$

\begin{tabular}{cccccc}
\hline Idade & \multicolumn{5}{c}{ Peso (kg) } \\
\cline { 2 - 6 } (anos) & $\mathbf{5 0}$ & $\mathbf{6 0}$ & $\mathbf{7 0}$ & $\mathbf{8 0}$ & $\mathbf{9 0}$ \\
\cline { 2 - 6 } & \multicolumn{5}{c}{ Homem } \\
\hline \multirow{2}{*}{20} & $3,7 * \mathrm{HR}-$ & $4,2 * \mathrm{HR}-$ & $4,7 * \mathrm{HR}-$ & $5,2 * \mathrm{HR}-$ & $5,6 * \mathrm{HR}-$ \\
& 201 & 238 & 273 & 307 & 339 \\
\multirow{2}{*}{30} & $3,6 * \mathrm{HR}-$ & $4,1 * \mathrm{HR}-$ & $4,6 * \mathrm{HR}-$ & $5,1 * \mathrm{HR}-$ & $5,5 * \mathrm{HR}-$ \\
& 197 & 233 & 268 & 301 & 333 \\
\multirow{2}{*}{40} & $3,5 * \mathrm{HR}-$ & $4,0 * \mathrm{HR}-$ & $4,5 * \mathrm{HR}-$ & $5,0 * \mathrm{HR}-$ & $5,4 * \mathrm{HR}-$ \\
& 192 & 228 & 262 & 295 & 319 \\
\hline
\end{tabular}

Fonte: Adaptado de ISO (2004).

Os valores encontrados em $\mathrm{W} / \mathrm{m}^{2}$ foram convertidos para $\mathrm{kcal} / \mathrm{h}$, conforme a fórmula 1 .

$$
\mathrm{kcal} / \mathrm{h}=W / \mathrm{m}^{2} * 1,54
$$

Em que: Kcal/h: carga de trabalho em kilocalorias/hora; e $\mathrm{W} / \mathrm{m}^{2}$ : carga de trabalho em Watts $/ \mathrm{m}^{2}$ (considerando $1,8 \mathrm{~m}^{2}$ de área corporal).

De forma complementar a esta análise, foi realizada por meio de regressão linear múltipla, análise das relações entre a carga física estimada e as variáveis pessoais: altura, peso e idade dos trabalhadores.

O segundo método, conhecido por carga cardiovascular de trabalho (CCV), um método proposto por Apud (1989) especificamente para o trabalho florestal e utilizado por diversos autores (ÇALIKAN \& ÇALAR, 2010; MINETTE et al., 2015). O método consiste basicamente na determinação de um limite de carga cardiovascular de um trabalhador para uma atividade laboral contínua, a partir da coleta de frequência cardíaca em diversas situações. Caso este limite seja ultrapassado, são calculadas pausas para descanso em minutos/hora de trabalho.

A carga cardiovascular do trabalho corresponde à porcentagem da frequência cardíaca durante o trabalho em relação à frequência cardíaca máxima suportada pelo trabalhador. Esta carga é calculada conforme a fórmula 2.

$$
C C V=\frac{F C T-F C R}{F C M-F C R} * 100
$$

Em que: CCV: carga cardiovascular (\%); FCT: frequência cardíaca de trabalho (bpm); FCM: freqüência cardíaca máxima (220 - idade); e FCR: freqüência cardíaca em repouso (bpm).

A frequência cardíaca limite ( $F C L$ ) em bpm (batidas por minuto) corresponde a $40 \%$ da carga cardiovascular máxima. A fórmula 3 a seguir.

$$
F C L=0,40 *(F C M-F C R)+F C R
$$

Em que: FCL: frequência cardíaca limite (bpm); FCM: freqüência cardíaca máxima (220 - idade); e FCR: freqüência cardíaca de repouso (bpm).

Quando a carga cardiovascular ultrapassa o valor de $40 \%$ é necessário reorganizar o regime trabalho/descanso, conforme a fórmula 4.

$$
\operatorname{Tr}=\frac{H t(F C T-F C L)}{F C T-F C R}
$$

Em que: Tr: pausa para descanso (minutos); $\mathrm{Ht}$ : tempo de trabalho avaliado (60 minutos); FCT: freqüência cardíaca de trabalho (bpm); FCR: freqüência cardíaca em repouso (bpm); e FCL: frequência cardíaca limite (bpm).

A partir da carga cardiovascular de trabalho, foi classificada a carga física, apresentada na Tabela 3.

Tabela 3. Classificação da carga física de trabalho por meio de frequência cardíaca para o método de Apud (1989)

\begin{tabular}{cc}
\hline $\begin{array}{c}\text { Frequência cardíaca média } \\
\text { (bpm) }\end{array}$ & $\begin{array}{c}\text { Classificação de carga de } \\
\text { trabalho }\end{array}$ \\
\hline$<75$ & Muito leve \\
$75,1-100$ & Leve \\
$100,1-125$ & Moderadamente pesada \\
$125,1-150$ & Pesada \\
$>150$ & Muito pesada \\
\hline
\end{tabular}

Fonte: Apud (1989). 


\section{RESULTADOS E DISCUSSÃO}

\section{Método do anexo C da norma ISO 8996}

As estimativas da carga física e as variáveis pessoais estão na Tabela 4.

Tabela 4. Estimativas da carga física de trabalho das ocupações analisadas, com uso do anexo $\mathrm{C}$ da norma ISO 8996

\begin{tabular}{|c|c|c|c|c|c|c|}
\hline Ocupação & Etapa & $\begin{array}{c}\text { Altura } \\
\text { (m) }\end{array}$ & $\begin{array}{l}\text { Peso } \\
(\mathrm{Kg})\end{array}$ & $\begin{array}{l}\text { Idade } \\
\text { (anos) }\end{array}$ & $\begin{array}{c}\text { HR } \\
\text { média } \\
\text { (bpm) }\end{array}$ & $\begin{array}{c}\text { Carga } \\
\text { trabalho } \\
\text { (kcal/h) }\end{array}$ \\
\hline $\begin{array}{c}\text { T1 } \\
\text { Cabeiro }\end{array}$ & $\begin{array}{c}\text { Pré- } \\
\text { arraste }\end{array}$ & 1,63 & 52 & 23 & 95 & 231,8 \\
\hline $\begin{array}{c}\text { T2 } \\
\text { Cabeiro }\end{array}$ & $\begin{array}{c}\text { Pré- } \\
\text { arraste }\end{array}$ & 1,86 & 90 & 23 & 102 & 357,6 \\
\hline $\begin{array}{c}\text { T3 } \\
\text { Cabeiro }\end{array}$ & $\begin{array}{c}\text { Pré- } \\
\text { arraste }\end{array}$ & 1,68 & 76 & 33 & 110 & 366,5 \\
\hline $\begin{array}{c}\text { T4 } \\
\text { Cabeiro }\end{array}$ & $\begin{array}{c}\text { Pré- } \\
\text { arraste }\end{array}$ & 1,75 & 63 & 28 & 120 & 398,9 \\
\hline Líder & Arraste & 1,68 & 60 & 36 & 84 & 171,6 \\
\hline Operador & Pátio & 1,80 & 75 & 38 & 104 & 317,2 \\
\hline
\end{tabular}

Foram verificados os possíveis efeitos dos aspectos pessoais na carga física de trabalho. Estas estimativas foram feitas com auxílio do anexo C da norma ISO 8996. Os resultados mostraram que a aplicação das variáveis peso, idade e frequência cardíaca média, resultaram em probabilidade altamente significativa com coeficiente de determinação $\left(R^{2}: 0,99\right)$, ou seja, estas variáveis explicam satisfatoriamente a variação da taxa metabólica.

Como a carga física do trabalho foi estimada a partir da frequência cardíaca média ( $p: 0,000$ ), foi esperada que esta variável tenha sido a mais influente para as estimativas. Esta variável representa a condição física e aeróbica do trabalhador, o que demonstra a importância de trabalhadores bem condicionados na atividade florestal, corroborando com Smith et al. (1985). Os autores Harstela (1990), Hagen et al. (1993) e Çalikan \& Çalar (2010) destacam que a frequência cardíaca é uma das variáveis mais eficazes para determinação da carga física e fadiga durante o trabalho florestal.

Dentre os 04 trabalhadores analisados para a mesma ocupação (puxador de cabo), houve diferença notável (42\%) de $167 \mathrm{kcal} / \mathrm{h}$ entre o trabalhador com maior (398 $\mathrm{kcal} / \mathrm{h}$ ) e menor gasto metabólico médio (231 kcal/h), fator que interferiu o desempenho, processo de fadiga e desidratação dos trabalhadores. Observou-se também que as médias de frequência cardíaca foram levemente maiores nas últimas horas da jornada de trabalho, fato também observado por Christie (2006) e relacionado à fadiga. A amplitude notável do gasto calórico por hora dentro da mesma ocupação (puxador de cabo) reitera a importância do condicionamento físico na exploração florestal, classificando o trabalho de moderado a pesado segundo a norma brasileira (BRASIL, 2014).

Estes resultados condizem com os encontrados por Batista (2008), que analisou a dieta dos trabalhadores do corte florestal na mesma área, concluindo que mesmo com o alto consumo energético na alimentação (4700 $\mathrm{kcal} / \mathrm{dia})$, os trabalhadores possuíam um índice de massa corporal considerado normal, o que é um forte indício da alta carga física imposta pela atividade. Essa constatação corrobora com os resultados de Sant'Anna \& Malinovski (2002), que definiram o perfil físico ideal de motosserristas, com base na grande variação de produtividade entre os mesmos.

\section{Método CCV (carga cardiovascular média)}

A Tabela 5 abaixo ilustra os resultados encontrados para as ocupações analisadas pelo método CCV\%.

Tabela 5. Variáveis pessoais dos trabalhadores avaliados e cálculos para mensuração do regime trabalho/descanso pelo método CCV\% de Apud (1989)

\begin{tabular}{ccccccc}
\hline Etapa & FCT & FCM & FCR & CCV & FCL & Tr \\
\hline Pré-arraste T1 & 114 & 197 & 72 & 33,6 & 122 & 0 \\
Pré-arraste T2 & 127 & 197 & 78 & 41,1 & 125,6 & 1,7 \\
Pré-arraste T3 & 136 & 187 & 79 & 52,7 & 122,2 & 14,5 \\
Pré-arraste T4 & 132 & 192 & 77 & 47,8 & 123 & 9,8 \\
Arraste & 94 & 184 & 71 & 20,3 & 116,2 & 0 \\
Pátio & 115 & 182 & 74 & 37,9 & 117,2 & 0 \\
\hline
\end{tabular}

Os resultados demonstraram que em três jornadas, todas referentes ao puxador de cabo da etapa de préarraste, os trabalhadores ultrapassaram o limite de $40 \%$ da carga cardiovascular, o que exige tempo de repouso (Tr) por hora de trabalho, em minutos. O maior regime de pausas encontrado definiu 14,5 minutos de descanso/hora. Houve grande variação na carga cardiovascular para a mesma ocupação nesta operação, entre $33,6 \%$ e $52,7 \%$, o que determinou carga física pesada para um trabalhador menos condicionado e carga moderada (sem necessidade de pausas) para outro melhor adaptado às condições.

Sanches (2012) verificou em seu estudo com trabalhadores da silvicultura e colheita, a exigência de 
pausas para descanso em todos os casos, com médias de $134 \mathrm{bpm}$ para os motosserristas. Estes resultados demonstram claramente a importância do condicionamento físico, citados por Smith et al. (1985) e Hagen et al. (1993), a consideração de características antropométricas na seleção de trabalhadores segundo Sant'Anna \& Malinovski (2002), os consumos periódicos de alimentos energéticos e água durante esta atividade (CHRISTIE, 2006; BATISTA, 2008).

\section{CONCLUSÃO}

A frequência cardíaca é uma boa indicadora de condicionamento físico, um aspecto importante a ser considerado durante o recrutamento de trabalhadores para a exploração florestal na Amazônia, pois interfere diretamente na saúde, segurança e desempenho durante o trabalho. Por fim, enfatizamos que se deve considerar a demanda energética e nutricional dos trabalhadores florestais na organização e elaboração das refeições diárias.

\section{AGRADECIMENTOS}

Gostaríamos de agradecer à equipe do Laboratório de Manejo Florestal (LMF-INPA) pela geração de ideias para enriquecimento da pesquisa na Amazônia. Por fim, a Mil Madeiras Preciosas Ltda pela concessão da área para pesquisa e por todo o suporte durante a estadia na empresa.

\section{DECLARAÇÃO DOS DIREITOS HUMANOS}

Todos os experimentos envolvidos neste estudo foram apreciados pelo comitê de ética do Instituto Nacional de Pesquisas da Amazônia, avaliados e aprovados pelo Comitê de Ética em Pesquisa (CEP) do Ministério da Saúde por meio da ferramenta eletrônica Plataforma Brasil.

\section{REFERÊNCIAS}

ALVARES, C.A. et al. Köppen's climate classification map for Brazil. Meteorologische Zeitschrift, v.22, p.1-18, 2013.

APUD, E. Human biological methods for ergonomics research in forestry. In: Guide-lines on ergonomic study in forestry. International Labour Organisation. Geneva, p.01-109, 1989.

BATISTA, H.L.D.P. Estudo de tempo e rendimento da motosserra considerando fatores ergonômicos numa exploração florestal na Amazônia Central. 2008. 105p. (Dissertação de mestrado).
BRASIL. Norma Regulamentadora № 15: atividades e operações insalubres. Brasília: Ministério do Trabalho e Emprego, Diário Oficial da União, 1978. Disponível em: <http://trabalho.gov.br/ index.php/seguranca-e-saude-notrabalho/normatizacao/normas -regulamentadoras>

CANZIAN, W.P. et al. Análise da carga física de trabalho em operações de colheita florestal semimecanizada em áreas declivosas. Revista Científica Eletrônica De Engenharia Florestal, v.22, p.09-17, 2013.

CHRISTIE, C.J. A field investigation of physical workloads imposed on harvesters in South African forestry. 2006. 193p. (Tese de doutorado).

ÇALISKAN, E.; ÇAGLAR, S. An assessment of physiological workload of forest workers in felling operations. African Journal of Biotechnology, v.9, p.5651-5658, 2010.

EMMERT, F. Combinação de dados de campo e métodos computacionais para o planejamento da exploração florestal na Amazônia. 2014. 190 p. (Tese de doutorado).

GARMIN. Garmin Connect. 2015. Disponível em: https://connect.garmin.com/pt-br

HAGEN, K.B. et al. Physical workload, perceived exertion and output of cut wood as related to age in motor-manual cutting. Ergonomics, v.36, p.479-488, 1993.

HARSTELA, P. Work postures and strain of workers in Nordic forest work: a selective review. International Journal of Industrial Ergonomics, v.5, p.219-226, 1990.

HIGUCHI, N. et al. Perspectivas do manejo florestal sustentável para a Amazônia brasileira. Hiléia - Revista do Direito Ambiental da Amazônia, v.5, p.78-93, 2007.

INMET. Banco de dados meteorológicos para ensino e pesquisa. Instituto Nacional de Meteorologia, 2016. Disponível em: <http://www.inmet.gov.br/portal/index.php?r=bdmep/ bdmep $>$

ISO 8996. Ergonomics of thermal environment - Determination of metabolic heat production. International Organization for Standardization, Geneva. 2004.

LEITÃO FILHO, H.F. Considerações sobre a florística de florestas tropicais e subtropicais do Brasil. IPEF, n.35, p.41-46, 1987.

LILLEY, R. et al. Survey of forest workers in New Zealand - Do hours of work, rest, and recovery play a role in accidents and injury? Journal of Safety Research, v.33, p.53-71, 2002.

LIMA, J.S.S.; LEITE, A.M.P. Mecanização In: MACHADO C.C. Colheita Florestal. 3. ed. Editora Viçosa, 2014. p.46-73.

MINETTE L.J. et al. Avaliação biomecânica e da carga física de trabalho dos trabalhadores florestais em regiões montanhosas. Scientia Forestalis, v.43, p.541-550, 2015.

SABOGAL, C. et al. Manejo florestal empresarial na Amazônia brasileira. Belém: CIFOR. 2006. 
SANCHES, A.L.P. Metas de produção em função da carga física do trabalho e repetitividade para operações de colheita florestal em terrenos montanhosos. 2012. 127p. (Dissertação de mestrado)

SANT'ANNA, C.M.; MALINOVSKI, J.R. Uso da análise multivariada no estudo de fatores humanos em operadores de motosserra. Cerne, v.8, p.98-104, 2002.

SMITH, L.A. et al. Heart-rate response to forest harvesting work in the South-Eastern United States during summer. Ergonomics, v.28, p.655-664, 1985. 